\title{
BRAF V600K Mutation Analysis
}

National Cancer Institute

\section{Source}

National Cancer Institute. BRAF V600K Mutation Analysis. NCI Thesaurus. Code $C 92465$.

A molecular diagnostic test performed to determine the presence or absence of a BRAF V600K mutation in a tissue sample. 